\title{
Effects of pump modulation on a four-level laser amplifier
}

\author{
Stephen H. Chakmakjian, Karl Koch, Stephanos Papademetriou, and C. R. Stroud, Jr. \\ The Institute of Optics, University of Rochester, Rochester, New York 14627
}

Received December 28, 1988; accepted May 23, 1989

\begin{abstract}
A theory is developed to describe the way in which modulations in the pump intensity produce modulations in the gain of a four-level, homogeneously broadened laser amplifier. The theory is tested by carrying out an experiment using an alexandrite crystal pumped by a cw dye laser. A second dye laser is used to measure the gain in the inverted laser transition. The dependence of the magnitude and the bandwidth of the gain on the pumping rate is determined. Agreement between theory and experiment is good.
\end{abstract}

\section{INTRODUCTION}

It has long been realized that population oscillations or pulsations play an important role in laser dynamics. ${ }^{1}$ By population oscillations we mean the modulation that occurs in the atomic inversion of a laser medium. Population oscillations can occur at the intermodal beat frequency of the laser and couple together different cavity modes. This coupling is important in the onset of mode locking as well as in the occurrence of laser instabilities.

This effect also occurs in homogeneously broadened absorbers. When a two-level absorber is pumped by a modulated beam the rate of absorption of the modulation sidebands depends on the modulation frequency. At low frequencies the absorption is decreased, producing a hole in the homogeneous line. ${ }^{2-8}$ This effect is the basis of amplitude modulation spectroscopy and has been studied experimentally in ruby, ${ }^{9}$ alexandrite, ${ }^{10}$ and fluorescein-doped glass. ${ }^{8}$

In this paper we will explore a closely related effect that has not been so well studied. We will see that population oscillations can also be produced by a modulated pump beam in a four-level laser medium. The modulation in the pump beam can modulate the gain for the laser field if the population can respond at the modulation frequency (see Fig. 1 for an energy-level diagram of a four-level laser system). This modulated gain can lead to the unstable operation of a laser. Ogawa and Hanamura ${ }^{11,12}$ and Ogawa ${ }^{13}$ have demonstrated that a modulated pump can produce instabilities in lasers.

In Section 2 we develop a rate-equation model of this effect for a canonical four-level system and derive an analytic expression for the stationary-state inversion as a function of modulation frequency and pumping rate. Then in Section 3 we describe an experimental test of this theory in alexandrite. The experiment is carried out using one dye laser as a pump to produce an inversion and a second, weak dye laser tuned to the inverted transition to probe the modulated inversion. In Sections 4 and 5 we compare the experimental results with theory.

Before we begin, it should be pointed out that the behavior of a laser is determined not solely by material properties but also by cavity configuration. If the cavity dynamics is included, the temporal response of the laser system to pump fluctuations can be slower than either the atomic dynamics or cavity dynamics alone would dictate. ${ }^{14}$ In this paper we concentrate on a laser-amplifier medium without an external cavity.

\section{THEORETICAL TREATMENT}

We present a theoretical analysis based on a canonical fourlevel system. This system is familiar to most laser researchers and is a commonly used model for many laser systems. Of course, no real laser is quite so simple as this model, but we can get a basic qualitative understanding of these effects by studying this simple four-level model.

We restrict our treatment to the case of strong collisional broadening. In this limit we are able to eliminate adiabatically the polarization equations of motion and start with the equations for the population density-matrix elements of the four-level system shown in Fig. 1:

$$
\begin{aligned}
& \dot{\rho}_{g g}=-\lambda \rho_{g g}+\gamma_{1} \rho_{11}, \\
& \dot{\rho}_{e e}=\lambda \rho_{g g}-\gamma_{e} \rho_{e e}, \\
& \dot{\rho}_{22}=\gamma_{e} \rho_{e e}-R\left(\rho_{22}-\rho_{11}\right)-\rho_{22} / T_{1},
\end{aligned}
$$

and

$$
\dot{\rho}_{11}=-\gamma_{1} \rho_{11}+R\left(\rho_{22}-\rho_{11}\right)+\rho_{22} / T_{1} .
$$

The ground-level population equation, Eq. (1a), contains the pump rate, $\lambda$, and the rapid nonoptical decay rate, $\gamma_{1}$. The equation of motion, Eq. (1b), for the population of the initial excited state contains the rapid nonoptical decay rate, $\gamma_{e}$. Finally, the spontaneous decay rate from level 2 is denoted by $1 / T_{1}$.

Since the nonoptical decays in the $\rho_{e e}$ and $\rho_{11}$ equations are rapid, we can adiabatically eliminate these variables from the system dynamics. When we do this and invoke conservation of population we are left with two independent equations:

$$
\dot{\rho}_{22}=-\left(R+\lambda+1 / T_{1}\right) \rho_{22}+\lambda
$$

and

$$
\dot{\rho}_{g g}=-\left(R+\lambda+1 / T_{1}\right) \rho_{g g}+R+1 / T_{1} .
$$

The factor $R+\lambda+1 / T_{1}$ appears as a homogeneous coefficient in both Eqs. (2a) and (2b). This term is the sum of the 


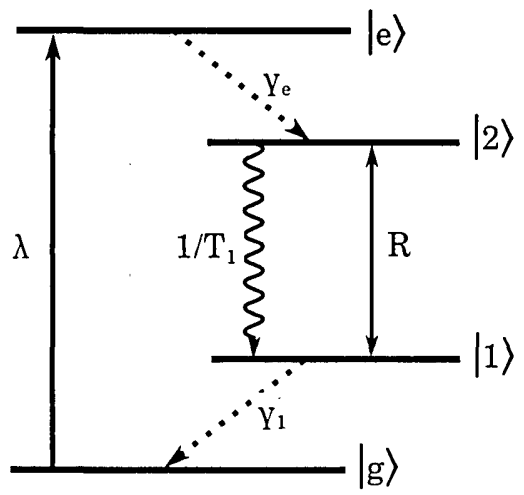

Fig. 1. Energy-level diagram for a canonical four-level laser system. Population is pumped from the ground state, $g$, to the initial excited state, $e$, by the pump rate, $\lambda$. The population rapidly decays from level $e$ to the upper lasing level, level 2, at a rate $\gamma_{e}$. The transition from level 2 to level 1 is naturally inverted if $\gamma_{1} \gg 1 / T_{1}$. Population is stimulated from level 2 to level 1 by the laser-field stimulated rate, $R$.

spontaneous-emission rate, $1 / T_{1}$, and the two stimulated rates, $R$ and $\lambda$; it is the population cycling rate, or rather the rate at which a population perturbation can circulate through the system's energy levels.

In the experiment we modulate the pump rate as

$$
\lambda(t)=\lambda_{0}+2 \delta \lambda \cos (\delta \omega t)
$$

where $\lambda_{0}$ is the dc or time-averaged pump rate, $\delta \lambda$ is the strength of the modulation ( $\delta \lambda \ll \lambda_{0}$ in this work), and $\delta \omega$ is the modulation frequency in radians per second.

Although we modulate only the pump laser, a modulation signal develops on the probe-laser stimulated rate, which we represent as

$$
R(t)=R_{0}+2 \delta R \cos (\delta \omega t+\Phi),
$$

where $\Phi$ is a phase lag that results from the finite response time of the atomic system. The modulation of the probe laser results from the modulated gain produced by the modulated pump rate, $\lambda(t)$. In general, because of nonlinearities, the resulting modulation on $R$ is anharmonic and contains higher harmonics of the modulation frequency, $\delta \omega$. However, since the modulation depth, $\delta \lambda$, is small in our experiment we neglect these higher-harmonic terms.

Floquet's theorem tells us that since the system is driven harmonically, the stationary-state response of the system will consist of harmonic overtones of the fundamental modulation frequency. The population for each of the levels in the system can be written as

$$
\rho_{j j}(t)=\sum_{n=-\infty}^{\infty} \rho_{j j, n} \exp (i n \delta \omega t),
$$

where $\rho_{j j, n}$ represents the $n$ th-harmonic component of the population response of energy level $j$.

We substitute the expressions for the time-dependent driving terms, Eqs. (3) and (4), and the expression for the time-dependent populations, Eq. (5), into the rate equations, Eqs. (2a) and (2b). When we equate terms of equal time dependence we get the following recurrence relations:

$$
\begin{aligned}
\left(I_{R}+I_{\lambda}\right. & \left.+1+i n \delta \omega T_{1}\right) \rho_{22, n}+\left(\delta I_{R}{ }^{*}+\delta I_{\lambda}\right) \rho_{22, n+1} \\
& +\left(\delta I_{R}+\delta I_{\lambda}\right) \rho_{22, n-1}=I_{\lambda} \delta_{n, 0}+\delta I_{\lambda}\left(\delta_{n, 1}+\delta_{n,-1}\right)
\end{aligned}
$$

and

$$
\begin{aligned}
\left(I_{R}+\right. & \left.I_{\lambda}+1+i n \delta \omega T_{1}\right) \rho_{g g, n}+\left(\delta I_{R}^{*}+\delta I_{\lambda}\right) \rho_{g g, n+1} \\
& +\left(\delta I_{R}+\delta I_{\lambda}\right) \rho_{g g, n-1}=\left(1+I_{R}\right) \delta_{n, 0}+\delta I_{R} \delta_{n, 1}+\delta I_{R}{ }^{*} \delta_{n,-1}
\end{aligned}
$$

where $I_{\lambda}$ and $I_{R}$ are the dimensionless intensities of the pump laser and the probe laser given by

$$
I_{\lambda}=\lambda_{0} T_{1}
$$

and

$$
I_{R}=R_{0} T_{1}
$$

The dimensionless intensities of the modulation sidebands are given by

$$
\delta I_{\lambda}=\delta \lambda T_{1}
$$

for the pump laser and

$$
\delta I_{R}=\delta R T_{1} \exp (i \phi)
$$

for the probe laser. The asterisks in Eqs. (6a) and (6b) denote the complex conjugate.

These recurrence relations can be solved iteratively to give an analytic solution for the population in terms of continued fractions. In this paper we consider the case when the modulation index is small $\left(\delta I_{\lambda}, \delta I_{R} \ll 1\right)$. This assumption allows us to truncate the continued fraction and obtain a solution to first order with respect to the modulation variables, $\delta I_{\lambda}$ and $\delta I_{R}$. The first-harmonic responses of the upper laser level (level 2) and the ground level (level g) in phase with the modulation are given by

$$
\operatorname{Re}\left(\rho_{22,1}\right)=\frac{\delta I_{\lambda}\left(1+I_{R}\right)-\delta I_{R} I_{\lambda}}{\left(1+I_{R}+I_{\lambda}\right)^{2}+\left(\delta \omega T_{1}\right)^{2}}
$$

and

$$
\operatorname{Re}\left(\rho_{g g, 1}\right)=\frac{\delta I_{R} I_{\lambda}-\delta I_{\lambda}\left(1+I_{R}\right)}{\left(1+I_{R}+I_{\lambda}\right)^{2}+\left(\delta \omega T_{1}\right)^{2}},
$$

respectively. Similarly, the atomic response $90^{\circ}$ out of phase with the modulation is given by the imaginary parts of $\rho_{22,1}$ and $\rho_{g g, 1}$. The response of the atomic population to a modulation of either stimulated rate, $R$ or $\lambda$, is a Lorentzian of half-width at half-maximum (HWHM) $\left(1+I_{\lambda}+I_{R}\right) / T_{1}$. In the limit of weak excitation $\left(I_{\lambda}, I_{R} \ll 1\right)$ the cycling rate is $1 / T_{1}$. These Lorentzian terms give rise to homogeneous hole burning or the $1 / T_{1}$ hole. ${ }^{6-10}$ The hole in the absorption spectrum of the modulation of the pump, $\delta \lambda$, arises from the negative term in Eq. (9b). The pump experiences decreased absorption when the population can follow the modulation frequency; another consequence of this is that a probe laser that is being amplified will experience a modulated amplification.

In the rate-equation limit we can write the propagation equation for the probe-laser intensity as

$$
\frac{\partial}{\partial z} I_{R}(t, z)=\alpha I_{R}(t, z) \rho_{22}(t, z)
$$




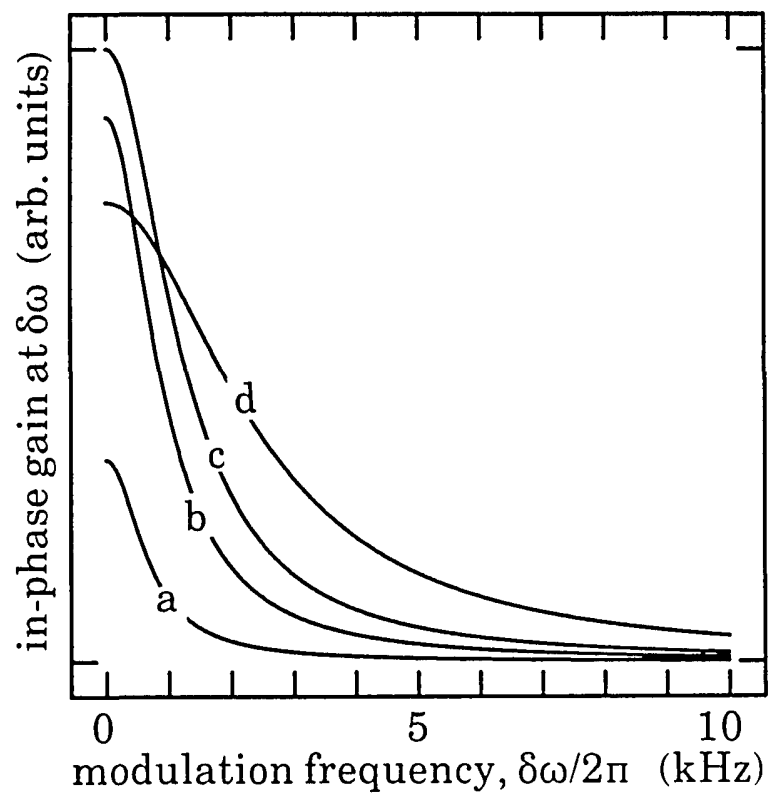

Fig. 2. In-phase linear gain, at the modulation frequency of the pump, experienced by a probe laser tuned to the inverted transition in a four-level system. The gain at the modulation frequency, $\delta \omega$, is plotted for weak probe-laser intensity $\left(I_{R} \ll 1\right)$. The gain is plotted for four values of the pump intensity: $\mathrm{a}, I_{\lambda}=0.1 ; \mathrm{b}, I_{\lambda}=0.5 ; \mathrm{c}, I_{\lambda}=$ $1.0 ; \mathrm{d}, I_{\lambda}=3.0$.

where $\alpha$ is the inverse Beer absorption length. Equating terms of equal time dependence, we get an expression for the spatial dependence of the probe field, $\delta I_{R}$ :

$$
\frac{\partial}{\partial z} \delta I_{R}(z)=\alpha\left\{\delta I_{R}(z) \rho_{22,0}(z)+I_{R}(z) \operatorname{Re}\left[\rho_{22,1}(z)\right]\right\} .
$$

The first term on the right-hand side of Eq. (11) is an exponential gain term, which is responsible for the growth of fields modulated at frequency $\delta \omega$. The exponential gain term is independent of modulation frequency for weak modulation. The second term on the right-hand side of Eq. (11) is a linear gain term. This linear gain term provides an avenue for pump fluctuations to be transmitted to the amplified probe-laser intensity, $I_{R}(t)$. In our experiment the exponential gain term is insignificant since the amplitude of the modulation sidebands, $\delta I_{R}$, remains small. It is this linear gain term that is responsible for the initial growth of the modulation signal in the intensity of the amplified probe laser. Furthermore it is the linear gain term that gives the amplifier a low-pass response to pump fluctuations. The physics of this behavior lies in the first-harmonic response of the excited-state population $\rho_{22,1}$. In our experiment we measure the response of an optical amplifier to amplitude fluctuations of the pump intensity, $I_{\lambda}(t)$. We have devised a signal-limited detection scheme to measure the spectrum of the amplifier's response to the fluctuations as a function of the pump modulation frequency, $\delta \omega$.

The results of Eq. (9a) are plotted in Fig. 2. We have plotted the real part of the first-harmonic component of the excited-state population, $\rho_{22,1}$, for various values of $I_{\lambda}$ and small probe-laser intensity $\left(I_{R} \ll 1\right)$. It is evident from the figure that the population behaves as a low-pass filter. The low frequency gain maximizes for $I_{\lambda}$ equal to one. The bandwidth of the gain increases with increasing pump inten- sity, $I_{\lambda}$, but the maximum gain at low frequency is obtained for $I_{\lambda}$ equal to $1+I_{R}$.

\section{EXPERIMENT}

We have carried out an experiment to test our theoretical predictions by measuring the amount of modulation transferred from a modulated pump laser to a probe laser being amplified in a four-level amplifier. The bandwidth of this modulation transfer is just the bandwidth of the first-harmonic response of the inverted level, $\rho_{22,1}$, which is expressed in Eq. (9a). When the modulation frequency is much greater than the population cycling rate the signal diminishes to zero. This detection scheme is signal limited in this respect. A diagram of the experimental setup is shown in Fig. 3.

The medium studied in our experiment is alexandrite. Alexandrite has an absorption maximum at $6000 \AA$. For this reason we use a Rhodamine $6 \mathrm{G}$ dye laser tuned to $6000 \AA$ as the pump laser. Alexandrite can be operated as a threelevel laser medium, like ruby, or as a four-level vibronic laser medium, like a dye. This system is described in the literature. ${ }^{15-17}$ In our experiment we tune the probe-laser frequency to the four-level laser transition of the alexandrite crystal. Although alexandrite is not an ideal four-level system, we have chosen this medium since both the pump and laser wavelengths are accessible with commercially available dyes. Furthermore, the population lifetime is long enough to probe with acousto-optic modulators available in our laboratory. In this experiment we tuned the probe laser, an LDS 698 dye laser, to the four-level or vibronic transition of alexandrite. The tuning range for this four-level operation is from 7000 to $8000 \AA$. We operate the LDS 698 dye laser at $7200 \AA$ in this experiment. Although this is not the center of the tuning curve of alexandrite, this wavelength is still visible to the eye, facilitating the alignment of the dye laser.

The Rhodamine laser beam passes through an acoustooptic modulator (AOM). We modulate the intensity of the zeroth-order or undiffracted beam by applying a modulated rf-drive voltage to the AOM. The strength of the modulation sidebands of the pump intensity, $\delta I_{\lambda}$, is kept near $10 \%$ of the time-averaged pump intensity, $I_{\lambda}$ (e.g., $\delta I_{\lambda} \simeq 0.1 I_{\lambda}$ ). The Rhodamine and LDS laser beams are combined by reflecting the Rhodamine beam off a dichroic mirror, which

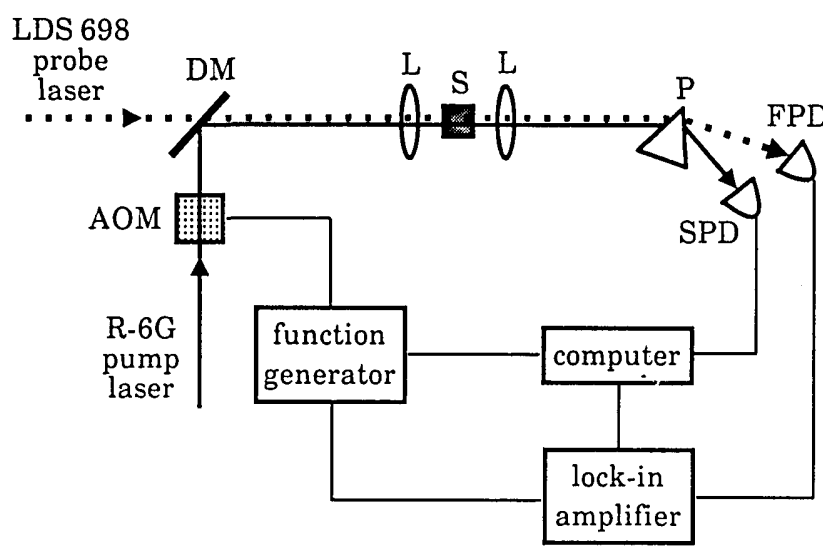

Fig. 3. Experimental apparatus in block-diagram form. We use the following abbreviations: AOM, acousto-optic modulator; DM, dichroic mirror; L's, lenses; S, sample; P, prism; SPD, slow photodiode; FPD, fast photodiode. 
transmits the LDS beam. The combined beams were focused into the alexandrite sample tightly enough to saturate the pump transition. Care was taken to ensure that the confocal parameter of the focused beams were longer than the sample. The alignment of the LDS laser was tweaked at this point to maximize the amplification signal, insuring maximum spatial overlap of the two laser beams. The laser beams were recollimated after leaving the crystal. A prism was used to separate the two laser beams spatially. The Rhodamine beam was directed onto a slow photodiode to measure the time-averaged intensity of the pump, $I_{\lambda}$. The LDS laser was directed onto a fast photodiode to measure the modulation signal in the intensity of the probe laser. The electrical signal from the fast diode was fed into a dualphase lock-in amplifier. The modulation signal driving the AOM was used as a reference signal to the lock-in amplifier.

The lock-in amplifier detected the amount of modulation transferred to the probe laser from the modulated pump, which creates a modulated gain in the alexandrite crystal. We used a microcomputer to control the modulation frequency driving the AOM by means of a voltage-controlled oscillator. The computer reads the two voltages from the two quadrature outputs of the lock-in amplifier. A data run consisted of sweeping the modulation frequency that is driving the AOM and recording the modulation frequency and the amount of modulation on the probe laser both in phase and in quadrature ( $90^{\circ}$ out of phase) with the modulation of the pump. Several data runs were taken at different pump intensities to study the effect of the pump intensity on the population cycling rate. A saturation measurement was made with the pump laser alone to determine the dimensionless intensity of this laser. The intensity determined from the saturation run was used in fitting the data.

The theory predicts that the bandwidth of the modulation transfer will broaden as the sum of the dimensionless intensities of the pump and the probe lasers. However, the cross section for the vibronic transition in alexandrite is an order of magnitude smaller than that of the pump transition; consequently we did not have adequate LDS laser intensity to broaden the bandwidth of the modulation transfer significantly with the probe laser.

\section{EXPERIMENTAL RESULTS}

In Fig. 4 we have plotted the in-phase modulation signal from the amplified probe laser. This form of our data is useful if one wishes to transfer information through an optical amplifier with no phase lag. This is good agreement between theory and experiment. Shown are results from four different pump-laser intensities. The data are fitted with the same theory. The free parameter used in the fitting process is the overall signal strength. The dimensionless intensity of the pump laser was derived from the saturation data.

We have plotted the in-quadrature modulation signal from the amplified probe in Fig. 5. Again we report good agreement between experiment and theory. These curves increase in size and width with increasing pump intensity. The in-quadrature signal behaves similarly to a dispersion curve. Since this response lags the in-phase response by $90^{\circ}$, the data and the theory are actually negative, but we have inverted them before plotting.

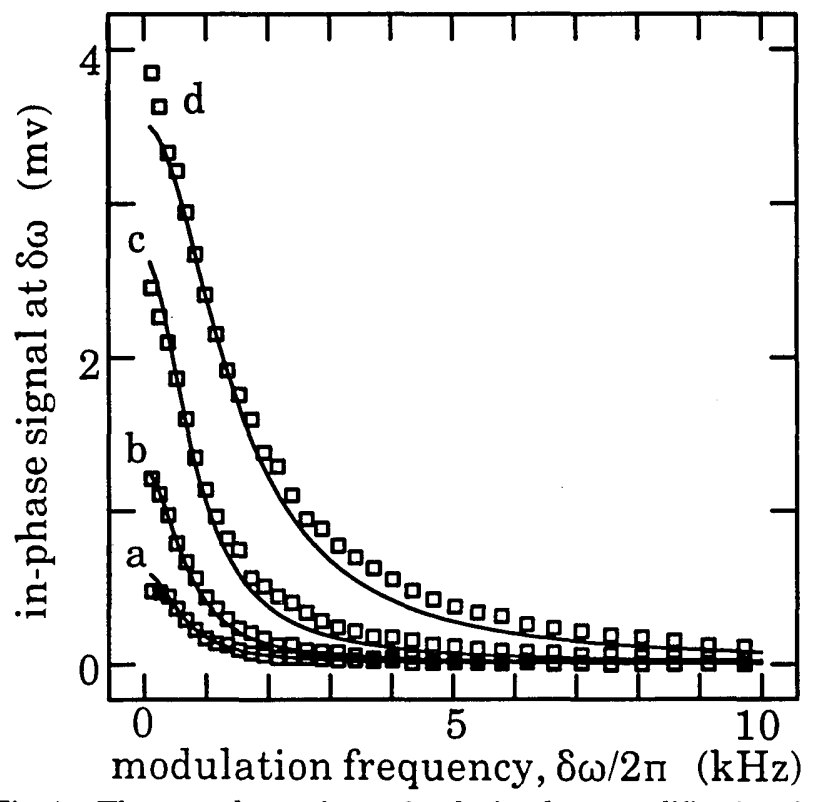

Fig. 4. Theory and experiment for the in-phase amplification signal at the modulation frequency $\delta \omega$. The squares represent data points and the solid curves the best theoretical fit using Eq. (9a). The in-phase signals are shown for negligible probe-laser intensity $\left(I_{R} \ll 1\right)$ and four different pump intensities: a, $I_{\lambda}=0.05 ; \mathrm{b}, I_{\lambda}=$ $0.12 ; \mathrm{c}, I_{\lambda}=0.37 ; \mathrm{d}, I_{\lambda}=1.5$.

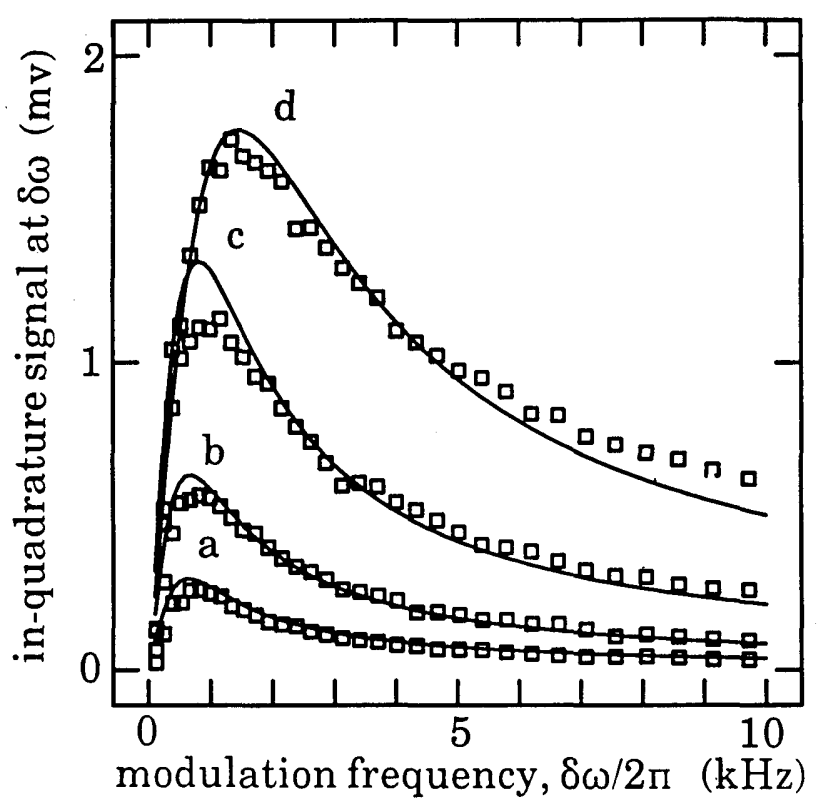

Fig. 5. Theory and experiment for the in-quadrature amplification signal at the modulation frequency $\delta \omega$. The squares represent data points and the solid curves the best theoretical fit. The signal is actually negative since we measure the modulation signal, which lags the modulation of the pump laser by $90^{\circ}$. With this in mind we have inverted the signal and the theory before plotting. Shown are the in-quadrature signals for weak probe-laser intensity and four different pump intensities: $\mathrm{a}, I_{\lambda}=0.05 ; \mathrm{b}, I_{\lambda}=0.12 ; \mathrm{c}, I_{\lambda}=0.37 ; \mathrm{d}, I_{\lambda}$ $=1.5$.

For applications to data communication the in-phase and in-quadrature data are of interest. If, however, the effect of multiplicative noise is the issue, then the phase of the modulation transfer is immaterial. The modulus of the modulation signal is analogous to the noise-transmission ability of 


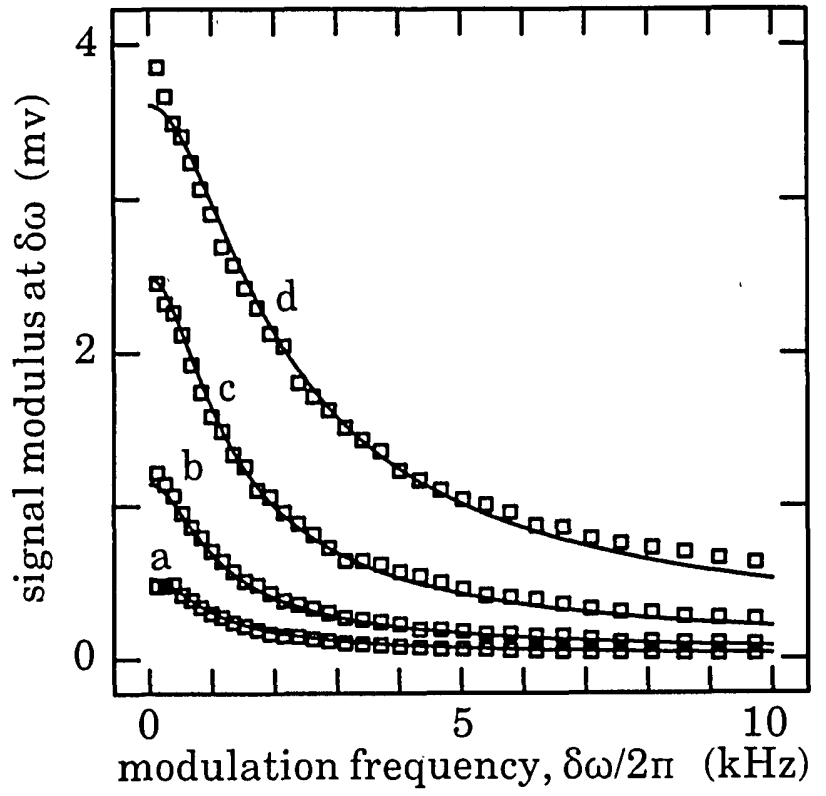

Fig. 6. Theory and experiment for the modulus of the amplification signal at the modulation frequency $\delta \omega$. The squares represent data points and the solid curves the best theoretical fit. The moduli of the amplification signals at $\delta \omega$ are plotted for negligible probelaser intensity $\left(I_{R} \ll 1\right)$ and four different pump intensities: a, $I_{\lambda}=$ $0.05 ; \mathrm{b}, I_{\lambda}=0.12 ; \mathrm{c}, I_{\lambda}=0.37 ; \mathrm{d}, I_{\lambda}=1.5$.

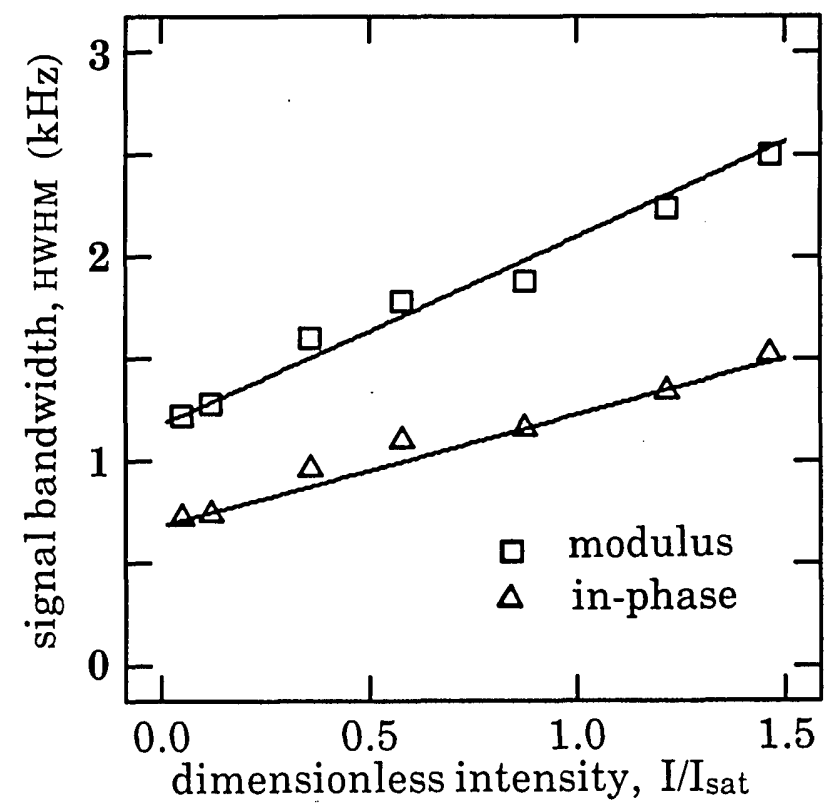

Fig. 7. HWHM versus the dimensionless intensity of the incoherent pump. Plotted is the HWHM of the in-phase signals and the HWHM of the signal modulus that we measure at the modulation frequency, $\delta \omega$, on the intensity of the probe laser. The HWHM of the in-phase data signal is represented as triangles, and the HWHM of the signal modulus is represented by the squares. The solid curves represent our theoretical predictions.

the system. ${ }^{14}$ In Fig. 6 we have plotted the modulus of the data (squares) and the theoretical value for the modulus (solid curves). The modulus signal increases with pump intensity and broadens, as did the in-phase signal. However the modulus falls off with increasing frequency more slowly than the in-phase signal does. The modulus behaves as the square root of the in-phase signal. The in-phase signal falls off as a Lorentzian; therefore the noise transmittance function of the amplifier is the square root of a Lorentzian.

A final interesting way to characterize the experiment is through the modulation bandwidth of the amplifier as a function of pump intensity. In Fig. 7 we have plotted the HWHM of the in-phase signal and the signal magnitude versus the dimensionless intensity of the pump laser. The bandwidth of the in-phase signal is always smaller than that of the signal modulus. Figure 7 shows that the bandwidth of the amplified signal increases with the dimensionless intensity of the pump laser. For the in-phase signal this increase is linear, and the slope is just the stimulated-emission rate of the pump laser, $I_{\lambda} / T_{1}$ (for alexandrite $T_{1}$ is the room-temperature fluorescence lifetime, $262 \mu \mathrm{sec}$ ). The $y$ intercept is the Einstein $A$ coefficient, $1 / T_{1}$.

\section{CONCLUSION}

In this paper we have studied the temporal response of a four-level laser amplifier to a modulated pump. Using a rate-equation formalism, which is valid for collisionally broadened homogeneous media, we have derived an expression for the response of the atomic population to a weak harmonic modulation of the pump rate of the amplifier. When the modulation sidebands are weak the response is a Lorentzian whose HWHM is the sum of the spontaneousemission rate and the dimensionless intensities of the pump laser, $I_{\lambda}$, and the probe laser, $I_{R}$ (the probe laser was tuned to the inverted transition). The HWHM is the rate at which population can cycle freely through the atomic system and is equal to $\left(1+I_{R}+I_{\lambda}\right) / T_{1}$. In the limit of weak driving $\left(I_{R}, I_{\lambda}\right.$ $\ll 1)$ the population responds at the spontaneous-emission rate, $1 / T_{1}$.

We have performed an experimental test of this result. By modulating the pump at a frequency $\delta \omega$ and measuring the amplification of the probe laser at the modulation frequency $\delta \omega$, we can measure the spectrum of the atomic response to the modulation. The probe laser undergoes modulated amplification only when the modulation frequency is less than the population cycling rate, or $\delta \omega<\left(1+I_{R}+I_{\lambda}\right) /$ $T_{1}$. Furthermore, we show that by measuring the modulus of the amplification signal one can measure the response of the amplifier to multiplicative pump noise.

The remarkable aspect of our results is that the intensity of the pump, $I_{\lambda}$, can affect the modulation bandwidth of the amplification that occurs between a different pair of atomic levels than the pumping process does. The pump is not coherent with the amplified probe, yet through population oscillations it has a marked effect on the modulation bandwidth of the amplifier.

The behavior and stability of a laser depend on both the atomic dynamics and the cavity configuration. We have shown that a proper treatment of the atomic dynamics must include the multilevel nature of the gain medium and the multifrequency nature of the field.

\section{ACKNOWLEDGMENT'S}

The authors acknowledge support of this research by the U.S. Army Research Office through the University Research Initiative Program and Allied-Signal Corporation. Also, we express gratitude to Jerzy Krasinski and Donald Harter for useful conversations. 


\section{REFERENCES AND NOTES}

1. M. Sargent III, M. O. Scully, and W. E. Lamb, Jr., Laser Physics (Addison-Wesley, Reading, Mass., 1974).

2. S. E. Schwarz and T. Y. Tan, "Wave interactions in saturable absorbers," Appl. Phys. Lett. 10, 4-6 (1967).

3. M. Sargent III, P. E. Toschek, and H. G. Danielmeyer, "Unidirectional saturation spectroscopy. I, Theory and short dipole lifetime limit," Appl. Phys. 11, 55-62 (1976).

4. M. Sargent III and P. E. Toschek, "Unidirectional saturation spectroscopy II," Appl. Phys. 11, 107-120 (1976).

5. L. W. Hillman, R. W. Boyd, and C. R. Stroud, Jr., "Natural modes for the analysis of optical bistability and laser instability," Opt. Lett. 7, 149-151 (1982).

6. R. W. Boyd, "Spectral holes due to population oscillations in a homogeneously broadened media," in Coherence and Quantum Optics V, L. Mandel and E. Wolf, eds. (Plenum, New York, 1984).

7. R. W. Boyd and S. Mukamel, "Origin of spectral holes in pumpprobe studies of homogeneously broadened lines," Phys. Rev. A 29, 1973-1983 (1984).

8. M. A. Kramer, R. W. Boyd, L. W. Hillman, and C. R. Stroud, Jr., "Propagation of modulated optical fields through saturableabsorbing media: a general theory of modulation spectroscopy," J. Opt. Soc. Am. B 2, 1444-1455 (1985).

9. L. W. Hillman, R. W. Boyd, J. Krasinski, and C. R. Stroud, Jr., "Observation of a spectral hole due to population oscillations in a homogeneously broadened optical absorption line," Opt. Commun. 45, 416-419 (1983).

10. M. S. Malcuit, R. W. Boyd, L. W. Hillman, J. Krasinski, and C. R. Stroud, Jr., "Saturation and inverse-saturation absorption line shapes in alexandrite," J. Opt. Soc. Am. B 1, 73-75 (1984).

11. T. Ogawa and E. Hanamura, "Dynamical properties of the multi-mode laser with modulated inversion," Opt. Commun. 61, 49-54 (1987).

12. T. Ogawa and E. Hanamura, "Numerical analysis of multimode laser with modulated inversion," Appl. Phys. B 43, 139153 (1987).

13. T. Ogawa, "Quasiperiodic instability and chaos in the bad-cavity laser with modulated inversion: numerical analysis of a Toda oscillator system," Phys. Rev. A 37, 4286-4302 (1988).

14. The effects of noise propagation from a pump laser to the secondary laser have been studied. This result includes cavity dynamics as well as material properties: A. W. Yu, G. P. Agrawal, and R. Roy, "Noise propagation from pump to secondary lasers," Opt. Lett. 12, 806-808 (1987).

15. J. C. Walling, O. G. Peterson, H. P. Jenssen, R. C. Morris, and E. W. O'Dell, "Tunable alexandrite lasers," IEEE J. Quantum Electron. QE-16, 1302-1314 (1980).

16. S. Guch, Jr., and C. E. Jones, "Alexandrite-laser performance at high temperature," Opt. Lett. 7, 608-610 (1982).

17. J. C. Walling, D. J. Heller, H. Samuelson, D. J. Harter, J. A. Pete, and R. C. Morris, "Tunable alexandrite lasers: development and performance," IEEE J. Quantum Electron. QE-21, 1568-1581 (1985). 